\title{
Assessing the effects of three potential chemical repellents to prevent bird damage to corn seeds and seedlings
}

Esther, A. ${ }^{1}$, Tilcher, R. ${ }^{2}$, Jacob, J. ${ }^{1}$

${ }^{1}$ Federal Research Institute for Cultivated Plants, Institute for Plant Protection in Horticulture and Forestry, Vertebrate Research, Toppheideweg 88, 48161 Münster, Germany, alexandra.esther@jki.bund.de

${ }^{2}$ KWS SAAT AG, Grimsehlstraße 31, 37555 Einbeck, Germany

DOI: $10.5073 /$ jka.2011.432.073

Bird damage to seeds and seedlings of corn and other crops is widespread in organic farming because no adequate seed protection is available. In this study we systematically tested the effect of seed treatments with three synthetically-produced substances known to affect bird feeding behavior (anthraquinone, pulegone and methylanthranilate) in different concentrations. There was no negative effect on the germination of seeds for all seed treatments. Their repellent effect was tested by food and seedling choice (treated versus untreated) experiments with pigeons (Columba livia) in aviaries. The best treatment variants in these trails were additionally tested regarding plant growth in the field where wild birds had unlimited access.

In aviaries, untreated seeds were clearly preferred over treated seeds by pigeons. The highest feeding deterrence effect occurred with the treatment variants $7.5 \%$ pulegone and $0.5 \%$ methylanthranilate. In these cases less than $5 \%$ of the daily consumption was treated seeds. In contrast, however, there was no treatment effect on seedlings. The same number of seedlings grown from treated and untreated seeds remained intact.

Further replications and field experiments with additional treatment variants are being conducted at the moment. The repellent effects of some of the treatment variants indicate that further studies with the same compounds but based on plants and benefit-cost-analyses should be done to yield a bird repellent to be used for organic seed protection. 